\title{
Segmented pulsed discharge for metastable argon lasing medium
}

\author{
Zhifan Zhang (张秩凡), Peng Lei (雷 鹏), Duluo Zuo (左都罗)”, and Xinbing Wang (王新兵) \\ Wuhan National Laboratory for Optoelectronics, Huazhong University of Science and Technology, Wuhan 430074, China \\ *Corresponding author: zuoduluo@hust.edu.cn \\ Received November 23, 2021 | Accepted December 29, 2021 | Posted Online January 24, 2022
}

\begin{abstract}
Direct current pulsed discharge is a promising route for producing high-density metastable particles required for optically pumped rare gas lasers [OPRGLS). Such metastable densities are easily realized in small discharge volumes at near atmospheric pressures, but problems appear when one is trying to achieve a large volume of plasma for high-power output. In this work, we examined the volume scalability of high-density metastable argon atoms by segmented discharge configuration. Two discharge zones attached with peaking capacitors were connected parallelly by thin wires, through which the peaking capacitors were charged and of which the inductance functioned as ballasting impendence to prevent discharging in only one zone. A uniform and dense plasma with the peak value of the number densities of $\operatorname{Ar}\left(1 \mathrm{~s}^{5}\right)$ on the order of $10^{13} \mathrm{~cm}^{-3}$ was readily achieved. The results demonstrated the feasibility of using segmented discharge for OPRGL development.
\end{abstract}

Keywords: rare gas laser; pulsed discharge; plasma application.

DOI: 10.3788/COL202220.031408

\section{Introduction}

High-power lasers are widely used in industrial and scientific fields. In recent years, solid-state lasers ${ }^{[1]}$, especially fiber lasers ${ }^{[2]}$, have been developed rapidly, but, due to the limitation of thermal self-focusing, facet damage, and non-linear effects ${ }^{[3]}$, the output power of a single fiber laser is difficult to increase further ${ }^{[4]}$. Diode pumped alkali lasers (DPALs) ${ }^{[5,6]}$ and optically pumped rare gas lasers (OPRGLs) ${ }^{[7]}$, which use gaseous lasing medium to avoid the bottleneck of solid-state lasers, are considered as potential new candidates for high-power lasers. The OPRGLs are kinetically analogous to the extensively studied DPALs, but overcome the disadvantages of DPALs by using a chemically inert lasing medium and offer the potential to achieve a specific laser power of the order of $10^{2} \mathrm{~W} \cdot \mathrm{cm}^{-3}$ with overall conversion efficiency as high as $60 \%{ }^{[8]}$. The OPRGLs are based on a three-level laser concept, including optical pumping of the $1 \mathrm{~s}^{5} \rightarrow 2 \mathrm{p}^{9}$ transition using a diode laser, collisional relaxation of the $2 \mathrm{p}^{9} \rightarrow 2 \mathrm{p}^{10}$ transition, and lasing through the $2 \mathrm{p}^{10} \rightarrow 1 \mathrm{~s}^{5}$ transition. The lower-level $1 \mathrm{~s}^{5}$ state is metastable, which is mainly generated by electric discharges. As an effective energy transfer agent, helium (He) is introduced into OPRGLs to near atmospheric pressure. In the past few years, progresses have been reported in OPRGLs, of which the laser power was increased from milliwatts to watts, and the optical conversion efficiency was increased from $0.1 \%$ to $31 \%^{[9-11]}$. The kinetic model $^{[12]}$ and experimental results ${ }^{[11]}$ showed that high density $\left(>1 \times 10^{12} \mathrm{~cm}^{-3}\right)$ of metastable atoms was necessary for efficient laser operation, but up till now this required particle density was only realized in a volume of less than $0.5 \mathrm{~cm}^{3}$ by direct current (DC) pulsed discharge at atmospheric pressure $^{[9]}$.

The main factor limiting the improvement of laser power is the volume of gain medium. The previous theoretical model suggested that a discharge volume of tens of cubic centimeters would be required for a $100 \mathrm{~kW}$ laser system ${ }^{[13]}$, which was much larger than the volume reported in the studies ${ }^{[9-11,14-19]}$. In fact, it is a common challenge of any electrical discharge to generate dense plasma in a large volume. The parallel electrode configuration in DC pulsed discharge offers the possibility of volume scaling-up. However, high-pressure discharges with large discharge gaps (to several centimeters) usually need complicated high-voltage pulsed power supply and pre-ionization devices and are limited to low repetition rates, which causes the result that the easiest way to enlarge the plasma volume may be the expanding of the electrode area. In our previous work $^{[20]}$, it was found that dense plasma could not be generated by using a pair of large plates in the pulsed discharge at high pressure. The inevitable thermal instability ${ }^{[21]}$ at atmospheric pressure seems to be the main reason for the difficulty of large-volume DC pulsed discharges with high repetition rates. Since this thermal instability is not serious under small-scale electrodes, inspired by the segmented excitation of high-power $\mathrm{CO}_{2}$ slab waveguide lasers ${ }^{[22,23]}$, we considered introducing a configuration of segmented discharge to provide large-volume 
and high-density lasing medium for OPRGLs. However, since each segmented electrode needs to be matched with a drive circuit, the design of the segmented discharge with separate driving circuits will be complicated. If all of the segmented electrodes are simply connected in parallel to the pulsed DC power supply, the expansion of the plasma volume cannot be realized in the nanosecond pulsed discharge. Because of this, the relative delay between the discharge current pulses passing through each segment is on the order of nanoseconds ${ }^{[24]}$, and the gas will possibly break down into a certain segment. Therefore, in order to reduce the complexity of the segmented pulsed discharge configuration, we consider connecting the peaking capacitors in parallel with each segmented electrode for the production of dense plasma. The peaking capacitors produce a narrow and intense current pulse, which enhance the ionization and radiation production at the first step of the discharge ${ }^{[25]}$. They are connected to a pulsed driving circuit by thin wires, of which the inductance functions as ballasting impendence to prevent discharging in only one segment. It is thus possible to increase the volume for the argon (Ar) metastable lasing medium by just increasing the number of segments.

\section{Experimental Setup}

A discharge chamber (with inner diameter $200 \mathrm{~mm}$ and height $400 \mathrm{~mm}$ ) of stainless steel six-way cross was constructed with four windows on the sides, which were anti-reflection coated for the IR radiation. A turbo pump (SL300, Leybold) at the top of the vacuum chamber was used to evacuate the chamber to $1 \times 10^{-6} \mathrm{mbar}(1 \mathrm{mbar}=101.325 \mathrm{~Pa})$ before filling with $\mathrm{Ar}$ and $\mathrm{He}$ gases. The pressure of the gas mixture ranged from 400 to $800 \mathrm{mbar}$, and the volume ratio of $\mathrm{Ar}$ and $\mathrm{He}$ was 1:99, which was expected to achieve the highest efficiency of excitation of Ar metastable states in $\mathrm{Ar} / \mathrm{He}$ mixtures ${ }^{[8]}$. As shown in Fig. 1, the discharge device was designed with a

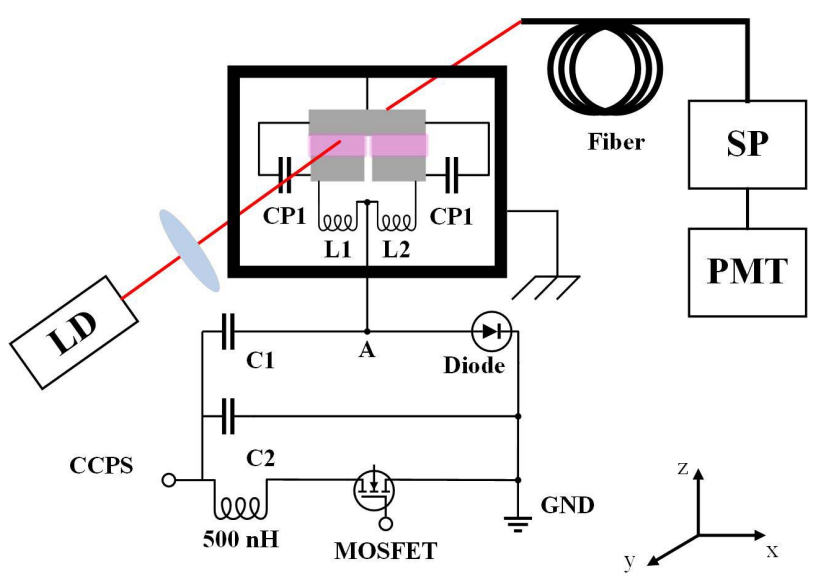

Fig. 1. Schematic of the plasma generator and the setup for laser diode absorption spectroscopy. LD, laser diode; SP, monochromator; PMT, photomultiplier tube; CCPS, capacitor charging power supply; MOSFET, metal-oxidesemiconductor field-effect transistor. segmented structure. All of the electrodes are made of stainless steel, and the upper one is electrically grounded. The area of each segmented lower electrode is $A=2.5 \times 0.8 \mathrm{~cm}^{2}$. The lower electrodes are parallel to the upper electrode and placed about $0.2 \mathrm{~cm}$ apart. The peaking capacitors (CP1 and $\mathrm{CP} 2)$ with a capacitance of $880 \mathrm{pF}$ each are connected in parallel with each segmented electrode pair in the discharge chamber and connected together at the high-voltage terminals by thin wires to the previously developed pulsed DC power supply ${ }^{[20]}$ via coaxial cable operating at $10 \mathrm{kHz}$. The inductance of L1 and L2 of the thin wires is estimated to be about $100 \mathrm{nH}$.

A home-made narrow linewidth $(10 \mathrm{GHz})$ diode laser ${ }^{[26]}$ was used for absorption spectroscopy to estimate the density of metastable atoms, and its power was attenuated to less than $1 \mathrm{~mW}$ by a neutral density attenuator. The setup and method are quite similar to those described in previous work ${ }^{[20]}$, so they are only briefly described here. The laser diode operating near $811.5 \mathrm{~nm}$ was aligned in the $y$ direction and loosely focused on the discharge gap. The beam passing through the plasma was collected by a photomultiplier tube (R928, Hamamatsu Photonics) through a monochromator (SP2750, Princeton Instruments). At the output of the pulsed DC power supply, the time-resolved waveforms of current and voltage were monitored by a current monitor (110, Pearson) and a high-voltage prober (10076B, Keysight), respectively, to observe the characteristics of segmented pulsed discharge.

\section{Experimental Results and Discussion}

The temporal waveforms of the voltage and current measured at point A marked in Fig. 1 are shown in Fig. 2. In the experiments, high-voltage pulses were adjusted by the voltage setting of a capacitor charging power supply (CCPS), and the maximum value of the pulsed voltage was slightly less than two times the charged voltage at the storage capacitors $\mathrm{C} 1$ and $\mathrm{C} 2$ with capacitance $4 \mathrm{nF}$ each. The related electric energy deposited into the plasma, calculated by integrating the product of the current

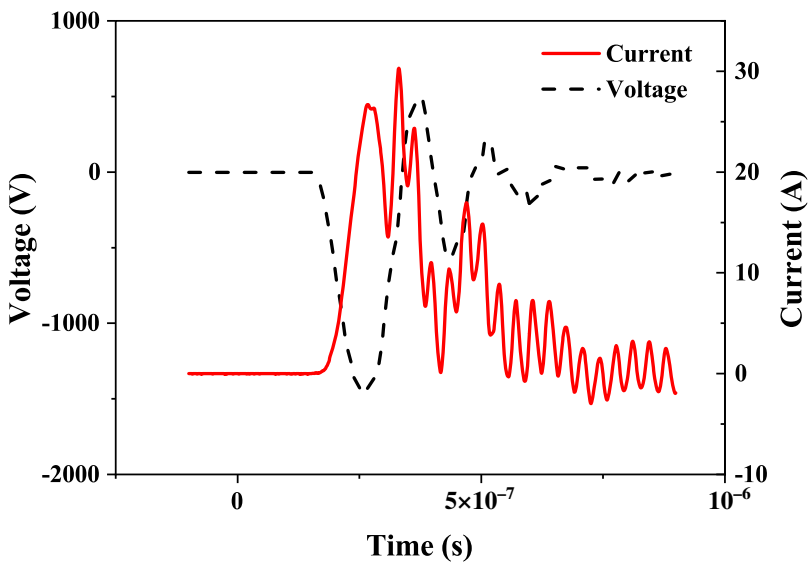

Fig. 2. Typical traces of pulsed voltage and current with gas mixture of $1 \% \mathrm{Ar} / \mathrm{He}$ at $800 \mathrm{mbar}$, of which the deposited energy is about $2.1 \mathrm{~mJ}$. CCPS is set at $800 \mathrm{~V}$, with the voltage at C1 and C2 charged to $1.33 \mathrm{kV}$. 
and voltage measured at point A, was about $2.1 \mathrm{~mJ}$. The current generated by the discharge device is one order of magnitude higher than that of Mikheyev et al. ${ }^{[15]}$, suggesting a higher degree of ionization in this case.

Photos of discharge plasma along the $x$ and $y$ directions are depicted in Fig. 3. The dense plasma uniformly filled the designed region, which demonstrated that the application of peaking capacitors reduced the effect of the jitter between the startup of each segment and produced dense plasma in two segments at the same time. The images of plasma were used for the estimation of the absorption length $l$ along the probing path. The transmitted laser intensity and the metastable density were calculated by the methods reported in the literature ${ }^{[11]}$.

In the experiments, we investigated the dependence of the density of metastable particles on the charging voltage by changing the voltage set at the CCPS, as shown in Fig. 4(a). The probing beam entered the plasma in a segment along the $y$ direction to avoid almost complete absorption by the plasma. We observed that the peak densities of metastable atoms produced in the experiments arrived at $1 \times 10^{13} \mathrm{~cm}^{-3}$, and it depended only slightly on the CCPS setting when the voltage was higher than $600 \mathrm{~V}$. This was a result of the breakdown voltages as well as the peak values of reduced electric field $E / N$ not changing much when higher charging voltages were applied. As shown in Fig. 4(b), the voltage waveform at point A of Fig. 1 was measured while the density of metastable atoms was measured. The output voltage of the pulsed DC power supply is highly dependent on the CCPS setting, so the gas mixture was not able to break down when its pressure was near atmospheric pressure, and the CCPS setting was lower than $500 \mathrm{~V}$. Because of the existence of cable inductance, there should be a process of resonant charging to the peaking capacitors, which made the minus peak of the discharge voltage at the lower electrode possibly even lower than that shown in Fig. 4(b).

High pressure is necessary for OPRGLs to improve the rates of relaxation between energy levels and broaden the absorption linewidth of metastable atoms for the pumping. However, it is noted that the breakdown voltage increases with the gas pressure, which affects the generation of metastable atoms. We investigated the effect of gas pressure on the generation of metastable atoms. As shown in Fig. 5, the peak value of the density of metastable atoms does not change significantly with the increase

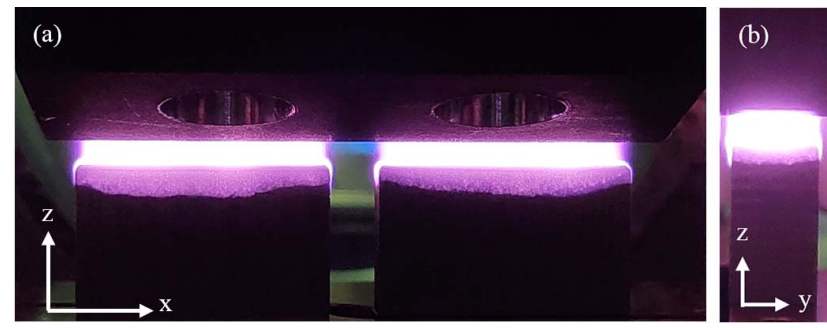

Fig. 3. Plasma photos taken along the (a) $y$ direction and (b) $x$ direction at $800 \mathrm{mbar}$. Two segmented electrodes were used each with a length of $25 \mathrm{~mm}$ in the $x$ direction and a width of $8 \mathrm{~mm}$ in the $y$ direction.
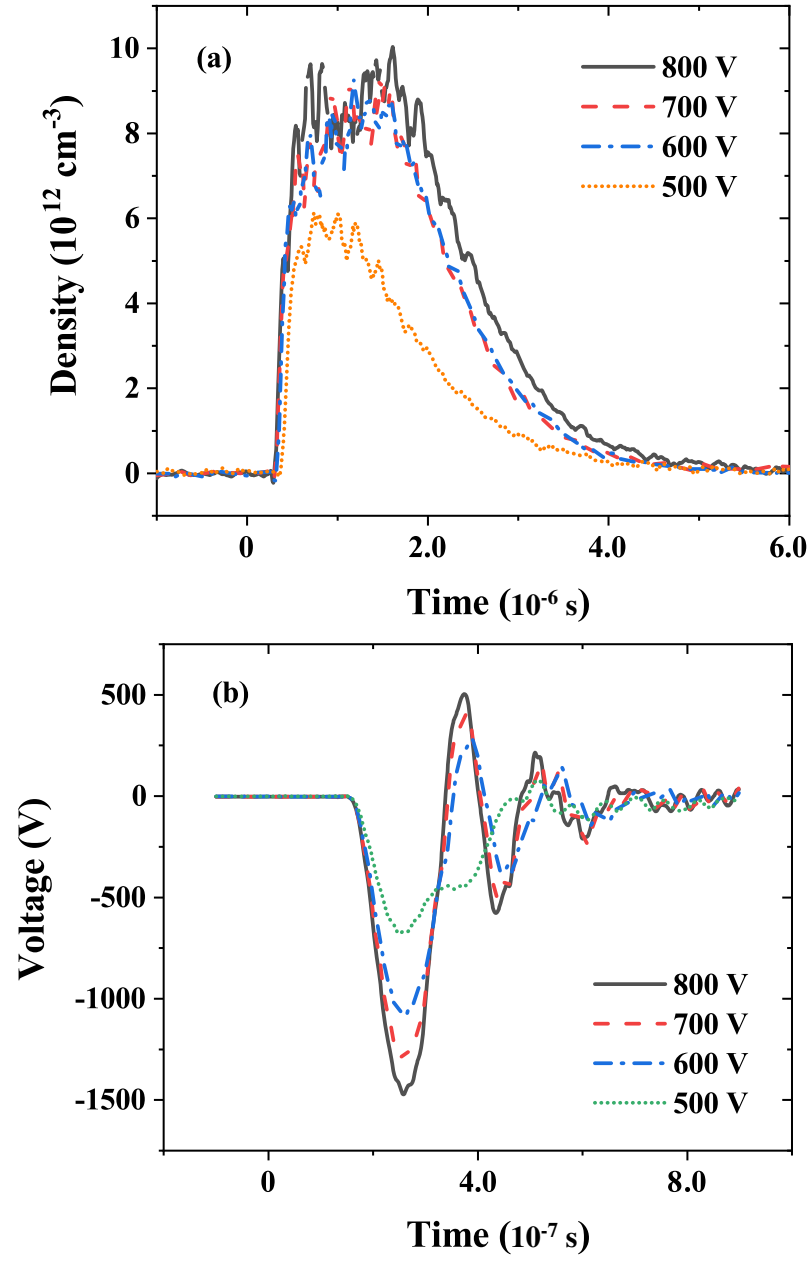

Fig. 4. (a) Curves of $\operatorname{Ar}\left(1 s^{5}\right)$ density versus time at different $D C$ charging voltages and 800 mbar. An absorption length of $8 \mathrm{~mm}$ was used. (b) Voltage at the output of pulsed DC power supply at different DC charging voltages and 800 mbar.

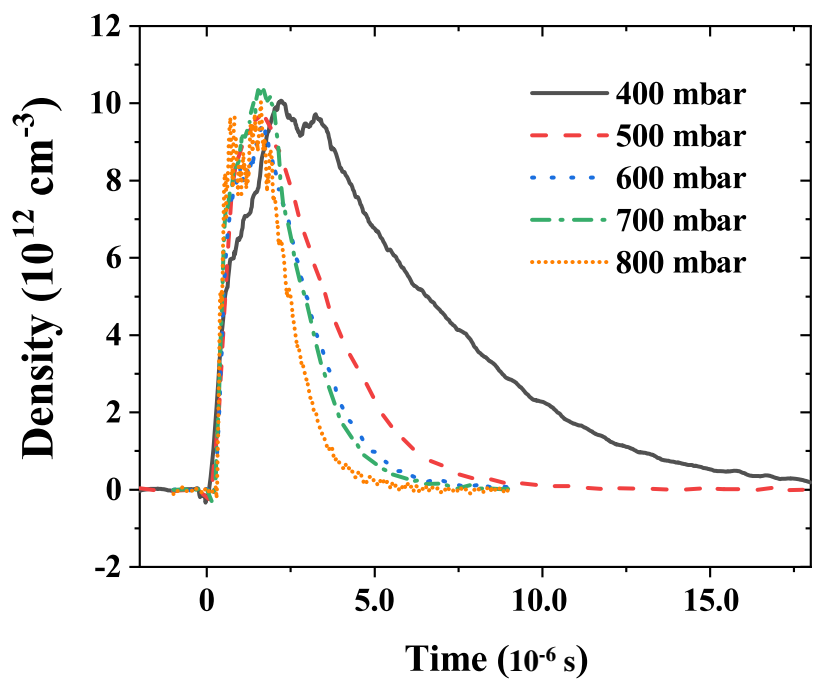

Fig. 5. Dependence of $\operatorname{Ar}\left(1 s^{5}\right)$ density on pressure. CCPS is set at $800 \mathrm{~V}$. An absorption length of $8 \mathrm{~mm}$ was used. 
Table 1. Wavelength and Transition Levels of Each Selected Line ${ }^{[27]}$.

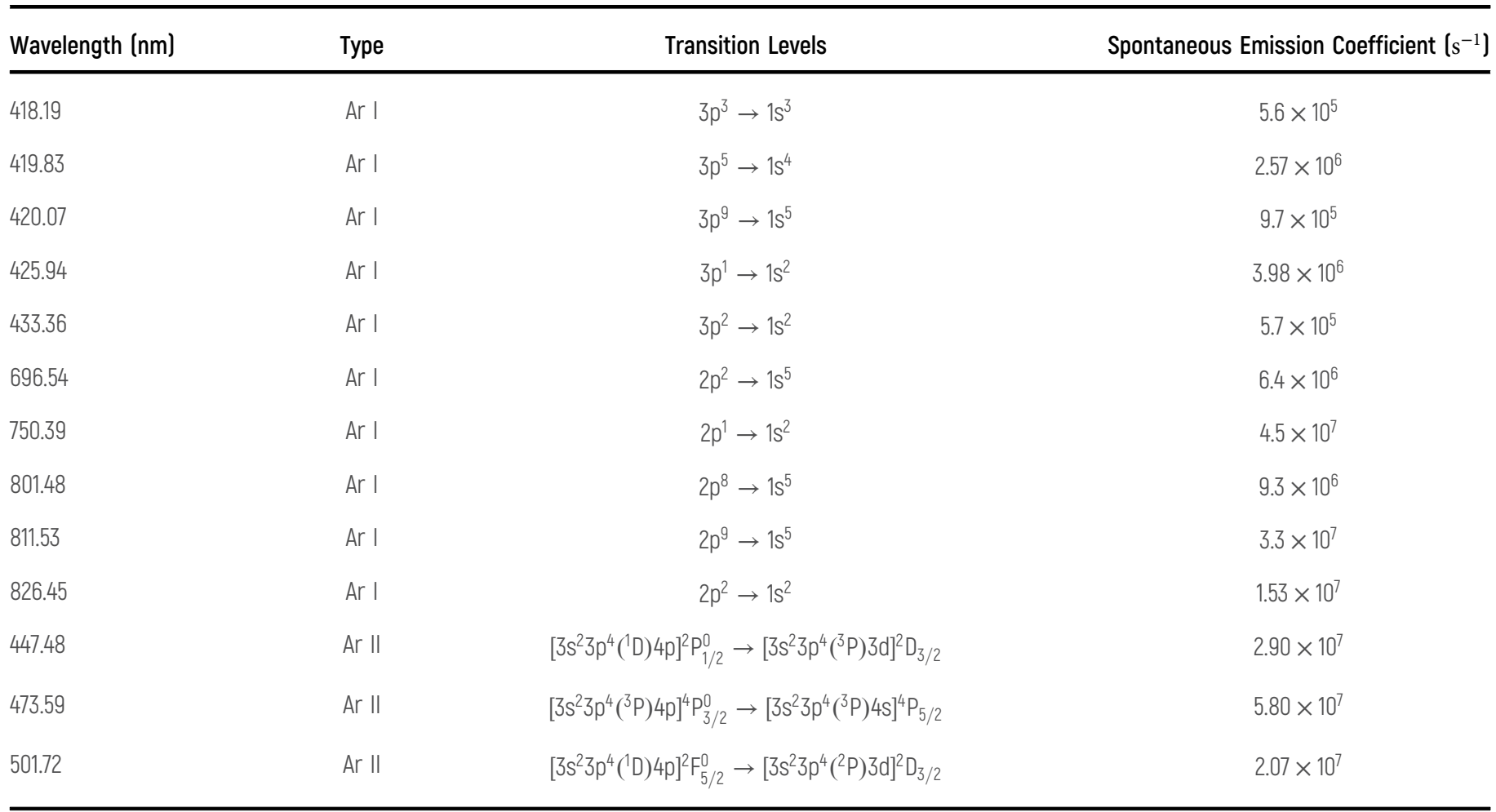
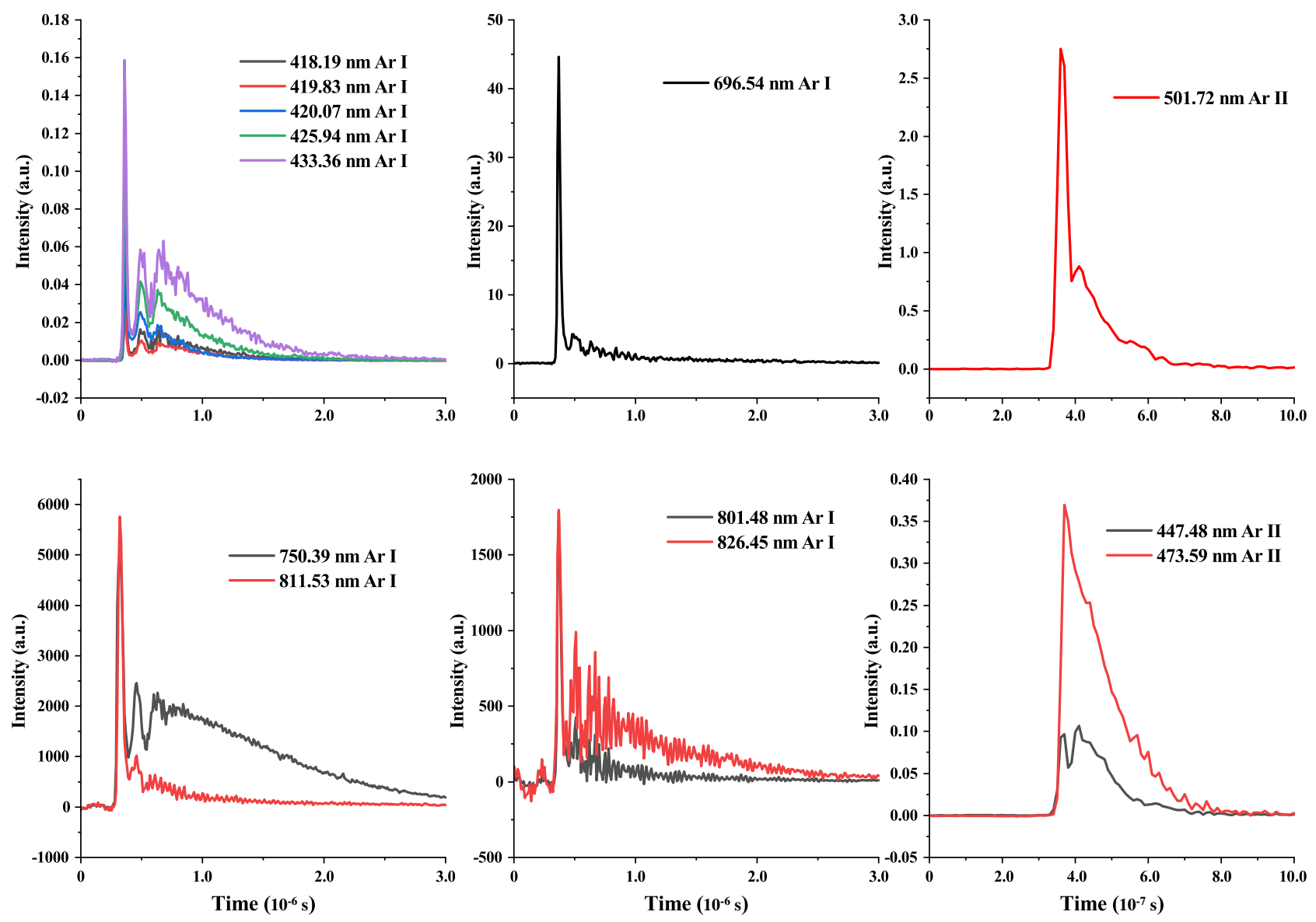

Fig. 6. Time-resolved intensity of characteristic emission of Ar atoms at $800 \mathrm{mbar}$. The DC charging voltage is $750 \mathrm{~V}$. 
of pressure, while the lifetime of metastable atoms decreases significantly with the increase of pressure. Therefore, high repetition rate [perhaps higher than $100 \mathrm{kHz}$, according to the duration of the metastable states shown in Fig. 4(a)] is required to continuously produce high-density metastable states in the segmented pulsed discharge at atmospheric pressure.

We investigated the temporal evolution of the characteristic emission lines of the gas mixture. The wavelength and related energy levels of the emission lines are shown in Table 1. Figure 6 shows that the temporal waveforms of emission lines exhibit a bimodal shape, indicating that the dominant mechanism leading to the generation of metastable atoms in such a pulsed discharge with high deposited energy is divided into two periods in time. Note that the pulsed current in Fig. 2 almost vanished in $1 \mu \mathrm{s}$ after the gas mixture got broken down. It can be seen from the temporal waveforms of different emission lines that a large number of active particles such as metastable Ar atoms, excited Ar atoms, and $\mathrm{Ar}$ ions were generated in the first duration of $100 \mathrm{~ns}$. After the pulsed current almost vanished, there was still afterglow, which indicated that when the electron excitation turned weak, the decay and relaxation collisions took the major role in the generation of metastable particles. In addition, since the intensity of emission is proportional to the occupation density of the upper level and the spontaneous emission coefficient, we noticed that the particle densities in the highly excited states of $\operatorname{Ar}\left(\right.$ such as $2 \mathrm{p}^{1}$ and $\left.2 \mathrm{p}^{2}\right)$, which were mainly produced by ion recombination and relaxation collision, were quite considerable compared to that of $2 \mathrm{p}^{9}$. Therefore, it is necessary to modify the pulsed discharge models ${ }^{[28,29]}$ used to simulate OPRGLs, which mainly investigate the excited states of $2 \mathrm{p}^{8}, 2 \mathrm{p}^{9}$, and $2 \mathrm{p}^{10}$ and simply use $\mathrm{Ar}(\mathrm{hl})$ to represent all energy levels of Ar above $2 \mathrm{p}^{8}$.

\section{Conclusions}

The primary objective of this study was to investigate the possibility of whether a segmented pulsed discharge could be used to realize a volume-scalable structure with high densities of $\operatorname{Ar}\left(1 s^{5}\right)$ metastable particles. This segmented discharge structure with peaking capacitors eliminated the need of separated drive circuits, reducing the complexity and cost of design. The peaking capacitors were charged by a DC pulsed power supply, and a narrow and intense pulsed current was generated every time when the discharge was triggered. Calculated from the measured voltage and current waveforms at the outlet of the pulsed power supply, the highest energy deposited into the discharge zone of $2 \times 0.4 \mathrm{~cm}^{3}$ was about $2.1 \mathrm{~mJ}$ for each pulse. The time-resolved results on metastable density and emission spectra showed that the lifetime of $\operatorname{Ar}\left(1 s^{5}\right)$ atoms was highly dependent on gas pressure, and the generation routes of $\operatorname{Ar}\left(15^{5}\right)$ atoms varied with time, where the ion recombination and the relaxation of highly excited particles took an important role at the afterglow stage. The peak metastable density approached $1 \times 10^{13} \mathrm{~cm}^{-3}$ at gas pressure of $800 \mathrm{mbar}$, which indicated that segmented pulsed discharge is a feasible scheme to generate scalable volume of high-density plasma at atmospheric pressure for Ar metastable production with applications in OPRGLs.

In fact, the practicability of this structure is far more than a one-dimensional arrangement. In subsequent experiments, the possibility of the two-dimensional array structure for further expanding the volume of dense plasma will be tested.

\section{References}

1. M. Zhao, C. Wang, Q. Hao, Z. Zou, J. Liu, X. Fan, and L. Su, "High singlepulse energy passively $Q$-switched laser based on $\mathrm{Yb}, \mathrm{Gd}: \mathrm{SrF}_{2}$ crystal," Chin. Opt. Lett. 18, 101401 (2020).

2. R. Lü, H. Teng, J. Zhu, Y. Yu, W. Liu, G. Chang, and Z. Wei, "High power $\mathrm{Yb}$-fiber laser amplifier based on nonlinear chirped-pulse amplification at a repetition rate of $1 \mathrm{MHz}$," Chin. Opt. Lett. 19, 091401 (2021).

3. H. Chen, X. Jiang, S. Xu, and H. Zhang, "Recent progress in multi-wavelength fiber lasers: principles, status, and challenges," Chin. Opt. Lett. 18, 041405 (2020).

4. J. W. Dawson, M. J. Messerly, R. J. Beach, M. Y. Shverdin, E. A. Stappaerts, A. K. Sridharan, P. H. Pax, J. E. Heebner, C. W. Siders, and C. P. J. Barty, "Analysis of the scalability of diffraction-limited fiber lasers and amplifiers to high average power," Opt. Express 16, 13240 (2008).

5. B. V. Zhdanov and R. J. Knize, "Review of alkali laser research and development," Opt. Eng. 52, 021010 (2013).

6. R. J. Beach, W. E. Krupke, V. K. Kanz, S. A. Payne, M. A. Dubinskii, and L. D. Merkle, "End-pumped continuous-wave alkali vapor lasers: experiment, model, and power scaling," J. Opt. Soc. Am. B 21, 2151 (2004).

7. J. Han and M. C. Heaven, "Gain and lasing of optically pumped metastable rare gas atoms," Opt. Lett. 37, 2157 (2012).

8. A. V. Demyanov, I. V. Kochetov, and P. A. Mikheyev, "Kinetic study of a cw optically pumped laser with metastable rare gas atoms produced in an electric discharge," J. Phys. D 46, 375202 (2013).

9. J. Han, M. C. Heaven, P. J. Moran, G. A. Pitz, E. M. Guild, C. R. Sanderson, and B. Hokr, "Demonstration of a CW diode-pumped Ar metastable laser operating at 4 W," Opt. Lett. 42, 4627 (2017).

10. P. J. Moran, N. P. Lockwood, M. A. Lange, D. A. Hostutler, E. M. Guild, M. R. Guy, J. E. McCord, and G. A. Pitz, "Plasma and laser kinetics and field emission from carbon nanotube fibers for an advanced noble gas laser (ANGL)," Proc. SPIE 9729, 97290C (2016).

11. W. T. Rawlins, K. L. Galbally-Kinney, S. J. Davis, A. R. Hoskinson, J. A. Hopwood, and M. C. Heaven, "Optically pumped microplasma rare gas laser,” Opt. Express 23, 4804 (2015).

12. Z. Yang, G. Yu, H. Wang, Q. Lu, and X. Xu, "Modeling of diode pumped metastable rare gas lasers," Opt. Express 23, 13823 (2015).

13. B. Eshel and G. P. Perram, "Five-level argon-helium discharge model for characterization of a diode-pumped rare-gas laser," J. Opt. Soc. Am. B 35, 164 (2018)

14. C. R. Sanderson, C. W. Ballmann, J. Han, A. B. Clark, B. H. Hokr, K. G. Xu, and M. C. Heaven, "Demonstration of a quasi-CW diode-pumped metastable xenon laser," Opt. Express 27, 36011 (2019).

15. P. A. Mikheyev, A. K. Chernyshov, M. I. Svistun, N. I. Ufimtsev, O. S. Kartamysheva, M. C. Heaven, and V. N. Azyazov, "Transversely optically pumped Ar:He laser with a pulsed-periodic discharge," Opt. Express 27, 38759 (2019).

16. H. Kim and J. Hopwood, "Scalable microplasma array for argon metastable lasing medium,” J. Appl. Phys. 126, 163301 (2019).

17. P. A. Mikheyev, J. Han, A. Clark, C. Sanderson, and M. C. Heaven, "Production of Ar and Xe metastables in rare gas mixtures in a dielectric barrier discharge," J. Phys. D 50, 485203 (2017).

18. A. R. Hoskinson, J. Gregório, J. Hopwood, K. L. Galbally-Kinney, S. J. Davis, and W. T. Rawlins, "Spatially resolved modeling and measurements of metastable argon atoms in argon-helium microplasmas," J. Appl. Phys. 121, 153302 (2017).

19. J. Han, L. Glebov, G. Venus, and M. C. Heaven, "Demonstration of a diodepumped metastable Ar laser,” Opt. Lett. 38, 5458 (2013). 
20. Z. Zhang, P. Lei, Z. Song, P. Sun, D. Zuo, and X. Wang, "Optically pumped argon metastable laser with repetitively pulsed discharge in a closed chamber,” J. Appl. Phys. 129, 143103 (2021).

21. A. Fridman, A. Chirokov, and A. Gutsol, "Non-thermal atmospheric pressure discharges," J. Phys. D 38, R1 (2005).

22. R. Chang and S. Shaw, "Compact high-efficiency $\mathrm{CO}_{2}$ slab waveguide lasers with segmented RF excitation," Jpn. J. Appl. Phys. 40, 4552 (2001).

23. J. D. Strohschein, W. D. Bilida, H. J. J. Seguin, and C. E. Capjack, "Enhancing discharge uniformity in a multi-kilowatt radio frequency excited $\mathrm{CO}_{2}$ slab laser array," Appl. Phys. Lett. 68, 1043 (1996).

24. E. A. Shershunova, V. Y. Khomich, M. V. Malashin, and S. I. Moshkunov, "Time-space radial development of nanosecond dielectric barrier discharge in flat air gaps under atmospheric pressure,” J. Phys. Conf. Ser. 982, 12014 (2018).
25. F. E. Sanz and J. M. G. Perez, "Peaking capacitor in an incomplete corona surface discharge preionized TEA $\mathrm{CO}_{2}$ laser," IEEE J. Quantum Electron. 27, 891 (1991)

26. J. Gao, D. Zuo, J. Zhao, B. Li, A. Yu, and X. Wang, "Stable 811.53 nm diode laser pump source for optically pumped metastable Ar laser," Opt. Laser Technol. 84, 48 (2016).

27. A. Kramida, Y. Ralchenko, J. Reader, and N. A. Team, NIST Atomic Spectra Database (ver. 5.8) (National Institute of Standards and Technology, 2020).

28. P. Sun, D. Zuo, P. A. Mikheyev, J. Han, and M. C. Heaven, "Time-dependent simulations of a CW pumped, pulsed DC discharge Ar metastable laser system," Opt. Express 27, 22289 (2019).

29. D. J. Emmons and D. E. Weeks, "Kinetics of high pressure argon-helium pulsed gas discharge," J. Appl. Phys. 121, 203301 (2017). 